Article

\title{
Decoupling Weather Influence from User Habits for an Optimal Electric Load Forecast System
}

\author{
Luca Massidda (D) and Marino Marrocu* \\ CRS4, Center for Advanced Studies, Research and Development in Sardinia, loc. Piscina Manna ed. 1, \\ 09010 Pula (CA), Italy; luca.massidda@crs4.it \\ * Correspondence: luca.massidda@crs4.it; Tel.: +39-070-9250-285
}

Received: 7 November 2017; Accepted: 12 December 2017; Published: 19 December 2017

\begin{abstract}
The balance between production and consumption in a smart grid with high penetration of renewable sources and in the presence of energy storage systems benefits from an accurate load prediction. A general approach to load forecasting is not possible because of the additional complication due to the increasing presence of distributed and usually unmeasured photovoltaic production. Various methods are proposed in the literature that can be classified into two classes: those that predict by separating the portion of load due to consumption habits from the part of production due to local weather conditions, and those that attempt to predict the load as a whole. The characteristic that should lead to a preference for one approach over another is obviously the percentage of penetration of distributed production. The study site discussed in this document is the grid of Borkum, an island located in the North Sea. The advantages in terms of reducing forecasting errors for the electrical load, which can be obtained by using weather information, are explained. In particular, when comparing the results of different approaches gradually introducing weather forecasts, it is clear that the correct functional dependency of production has to be taken into account in order to obtain maximum yield from the available information. Where possible, this approach can significantly improve the quality of the forecasts, which in turn can improve the balance of a network-especially if energy storage systems are in place.
\end{abstract}

Keywords: net load forecasting; high renewable penetration; microgrid; renewable energy integration

\section{Introduction}

The integration of renewable energy generation has introduced a new strong variability and intermittence in the power grids, making it increasingly difficult for utilities to maintain the balance between energy production and consumption [1,2].

Energy storage and generation forecasts are the most promising strategies to reduce the negative impact of the variability of renewable energy production for a small grid, particularly for use on an island [3]. These strategies are one of the topics studied in the European H2020 NETfficient project (http:/ / www.netfficient-project.eu), to which this work is related. The project aims to propose an effective innovation in the use and distribution of electric power through the development of several energy storage technologies and an energy management system, enabling the implementation of a smart grid on the German island of Borkum in the North Sea.

Battery energy storage systems (BESS) can mitigate the variability and uncertainty associated with renewable resources and can increase the share of renewable energy that can be introduced into the distribution grid $[1,4,5]$. The balance of the electrical network and the optimal control of the accumulation system passes through an accurate forecast of load and generation in the network. Load prediction is essential for the efficient and reliable operation of power supply systems, leading to continuous consumer power supply [6]. 
In the literature, load forecasts are classified according to the time horizon [7]: the long-term forecast has a horizon of one to ten years and is required for planning investments and maintenance; medium-term forecasting has a horizon in the range of one week to a month, and is used to schedule fuel supply and to schedule maintenance activities; short-term forecasting refers to times ranging from one hour to one week, and is used for economic optimization, by scheduling loads and maintenance; very short-term load forecasting refers to prediction times ranging from seconds to hours, and is responsible for maintaining the balance between demand and power generation.

Several meta-review papers provide a good overview of the relevant literature [8-13].

This paper is focused on a short-term forecast, with a forecast horizon of two days and a resolution of $1 \mathrm{~h}$. Short-term forecasting techniques can be divided into classical statistics techniques and machine-learning methods.

Classical techniques include: regression models, auto regressive models (ARIMA) [14], time series models [15,16], Kalman filtering methods [17], and double seasonal Holt-Winters exponential smoothing (DSHW) [18], among others. These models perform satisfactorily under normal conditions, but do not offer adequate performance in the face of sudden changes. Furthermore, errors in the predictions provided by these methods increase considerably due to the influence of exogenous (human impact, social and cultural events) and meteorological (i.e., relative humidity of temperature and wind speed) variables.

In recent years, machine learning and artificial intelligence (AI) forecasting technology have been gradually replacing the classical techniques. A recent review reports the more significant benefits of AI methods with respect to the purely statistical ones, and shows the increasing use of hybrid methods [12].

Neural network approaches combined with other methods (such as evolutionary or fuzzy methods) are most frequently used [7,19-24], and a lot of interest is now reserved for deep learning, which seems very promising and uses multi-layered neural networks (e.g., convolutional or recurrent neural networks) [25].

Proposed by Vapnik [26], support vector machine regression (SVR) is another machine learning method which is widely used and has been studied for short-term power load forecasting.

This class of algorithms uses a high-dimensional feature space via nonlinear mapping through a kernel function, and performs a linear regression in that space. Thus, the nonlinear problem in the original lower-dimensional input space could find its linear solution in the higher-dimensional feature space.

Compared to the neural network algorithm, SVR has the advantages of robustness, high generalization ability, fast computation speed, and the capacity to avoid falling into local optima [27]; it requires a smaller training data set [28] and is widely used and studied for short-term power load forecasting $[7,29,30]$.

Currently, the high penetration of solar and wind generation adds further uncertainty to the forecast of loads.

The energy production of photovoltaic (PV) plants and, even more, of wind turbines is closely linked to weather conditions, and this entails a further difficulty in forecasting power generation and grid net consumption.

Improving the power forecast of wind turbines and photovoltaic systems is essential for increasing their share in the energy mix and for optimally sizing the storage capacity required for grid stability. To this end, an accurate forecast is essential, as is knowing the uncertainty associated with that prediction [31].

It is difficult to compare the many different techniques that have been developed over the years; this is due to the peculiarities of each application, which depend on the type of installation, geographic characteristics, time horizons of interest, and also on the purpose for which a forecast is required. The estimated power production is the output of the representative model of the wind turbine or of 
the PV plant, which according to the forecasting technique used, can receive different types of weather variables as input, which in turn are the output of some Numerical Weather Prediction (NWP) models.

A recent and complete review of the techniques proposed in the literature can be found in [32] for wind turbine power forecasting and in [33] for PV plants.

The ever-increasing spread of domestic photovoltaic plants poses the problem of a load forecast net of distributed generation, as these PV systems do not always have a dedicated meter, and it is nonetheless difficult to process the big data resulting from such meters.

Given that generation from renewable sources has a higher variance than the simple load, forecasting net load in the presence of a large distributed generation is more difficult to achieve, and traditional load forecasting systems can become less and less efficient as the power generated by PV systems increases [34].

The net load forecast for a distribution network will necessarily be directly linked to weather conditions such as irradiation or cloud coverage, which determine the production of PV systems [35].

The net load forecast also occurs in the case of microgrids to evaluate the exchanges with the distribution network, the balance of the grid itself when it is isolated [1], and for the optimal control of any storage system in the grid [36]. In this case, it is possible that medium-sized PV plants and wind turbines may be connected to the microgrid, and the net power exchange of the grid will be even more influenced by the meteorological variables.

Efforts to improve net load forecasts have been growing in recent years.

Some recent papers have proposed an integrated approach to achieve a direct forecast of the net load of a distribution network [1] and the power exchange of a micro-grid [24,37,38]. In this case, the regression model uses the forecasts of the main meteorological variables of interest as input variables in addition to the predictors commonly used in traditional load forecasting, such as historical data, the so-called calendar effects (such as the time of day, the day of the week, the public holidays), or other social factors.

Some papers take advantage of the measurements of distributed generation through smart meters and of the total load to make a separate forecast of production and consumption, and thus of the net load [38,39].

Finally, in some works (such as this one), attempts are made to deduce and separate the renewable energy generation from load measurement. In this case, the net load forecast is obtained from a reduced set of measurements, through knowledge or estimation of installed production capacity [40-42].

The work aims to analyze the performance of a forecast system for the load of the distribution grid and of a forecast system for the power exchange between the microgrid of the island and the power grid on the mainland. The forecast has a resolution of one hour and a two-day horizon to ensure that it could cover at least $24 \mathrm{~h}$ and be issued at least one day in advance of its use.

The paper will highlight:

- the importance of an accurate forecast of photovoltaic output for low-voltage load estimation;

- how an estimate of the installed photovoltaic capacity can be inferred from the measured net power consumption and meteorological information;

- how - at least in this case - the use of separate models for the prediction of individual plants can yield better results than those obtainable by training a single regression model for the microgrid.

The remainder of the paper is organized as follows. In Section 2 the available data for the power measurements and meteorological data are described. In Section 3 the forecast approaches adopted for net load forecast and power exchange are illustrated. Section 4 contains the discussion of the results obtained, and finally in Section 5 the conclusions and future directions are drawn. 


\section{Data}

\subsection{Borkum Grid}

Borkum Island lies in the North Sea, and is connected to the mainland via $20 \mathrm{kV}$ cables (network operator EWE NETZ GmbH, Oldenburg). On the island, power is transformed via 60 local substations from $20 \mathrm{kV}$ to $400 \mathrm{~V}$ and delivered via the low-voltage (LV) distribution networks to the households.

The scheme in Figure 1 shows the overall component architecture of the grid design. In the medium-voltage (MV) grid are two wind turbines, a PV power plant, and three emergency generators (they get only activated when the connection to the mainland is interrupted); the figure also shows the MV BESS realized in the NETfficient project. The two identical wind turbines are located in the south-west of the island, close to the sea, and have a rated power of $1800 \mathrm{~kW}$ each. The MV PV park is located in the middle of the island, and has a nominal power of $1387 \mathrm{kWp}$.

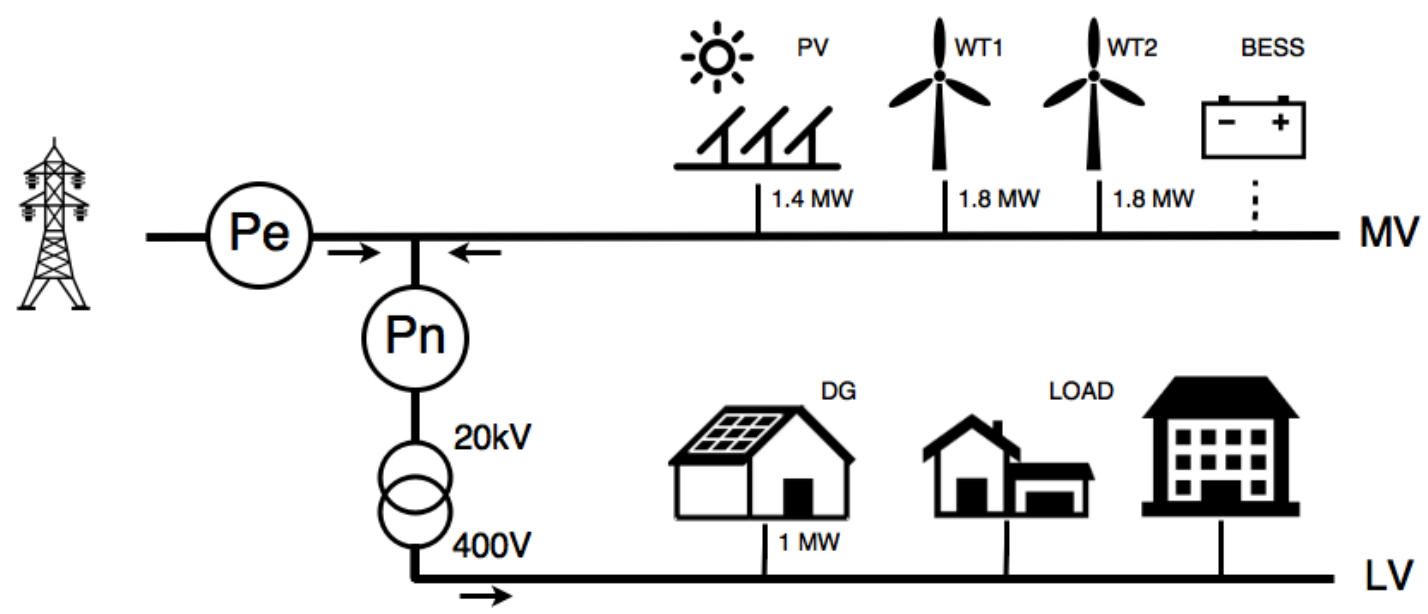

Figure 1. Scheme of the grid in Borkum. A photovoltaic (PV) power plant and two wind turbines are connected to the medium-voltage (MV) line. An energy storage system will be connected to the MV line in the future. The low-voltage (LV) line distributes power to the town, where several PV devices are connected. The available power measurements are relative to the total power exchange with the mainland $\left(p_{e}\right)$ and to the LV net load $\left(p_{n}\right)$. BESS: battery energy storage system.

There are are $5600 \mathrm{~m}$ connected to the LV serving a population of 5200, three industrial CHP (combined heat and power), seven home CHP and, 101 PV devices with a total PV capacity close to $1 \mathrm{MW}$. Tourism is essential for the island's economy, and the number of residents increases considerably during holidays and summer.

The net power exchange with the mainland and the net load of the LV distribution have been measured with a frequency of $15 \mathrm{~min}$. The available measures are relative to the years 2012, 2013, and 2014. The data has been averaged over a period of $1 \mathrm{~h}$ for this analysis. The data of the first two years will be used as the training set for the regression models; the testing will be done on the measures of the year 2014.

Table 1 summarizes the properties of the available power measurements. Figure 2 shows the box plots of the net load on the low-voltage distribution grid and of the power exchange of the microgrid as a function of the hour of the day. Two peaks are evident in both plots-during the morning and the evening. The variability is maximum during daytime for the net load, and is minimum at nighttime. Both the local minimum near noon and the high variability during daytime are partially related to the distributed PV generation. The variability of the power exchange is approximately constant during the day. This is related to the wind turbine production, which does not have any daily pattern. 
Table 1. Number of samples for power measures available for the Borkum grid. Three years, with $15 \mathrm{~min}$ frequency, of power measures in two measurement points are available. The minimum, maximum, mean values, and the standard deviations are listed.

\begin{tabular}{ccc}
\hline & Power Exchange $\boldsymbol{p}_{\boldsymbol{e}}$ & Net Load $\boldsymbol{p}_{\boldsymbol{n}}$ \\
\hline number & 105,216 & 105,216 \\
mean $(\mathrm{kW})$ & 2029.5 & 3603.7 \\
std $(\mathrm{kW})$ & 1600.7 & 1137.1 \\
$\min (\mathrm{kW})$ & -2303.6 & 1446.2 \\
$\max (\mathrm{kW})$ & 6651.2 & 6928.9 \\
\hline
\end{tabular}
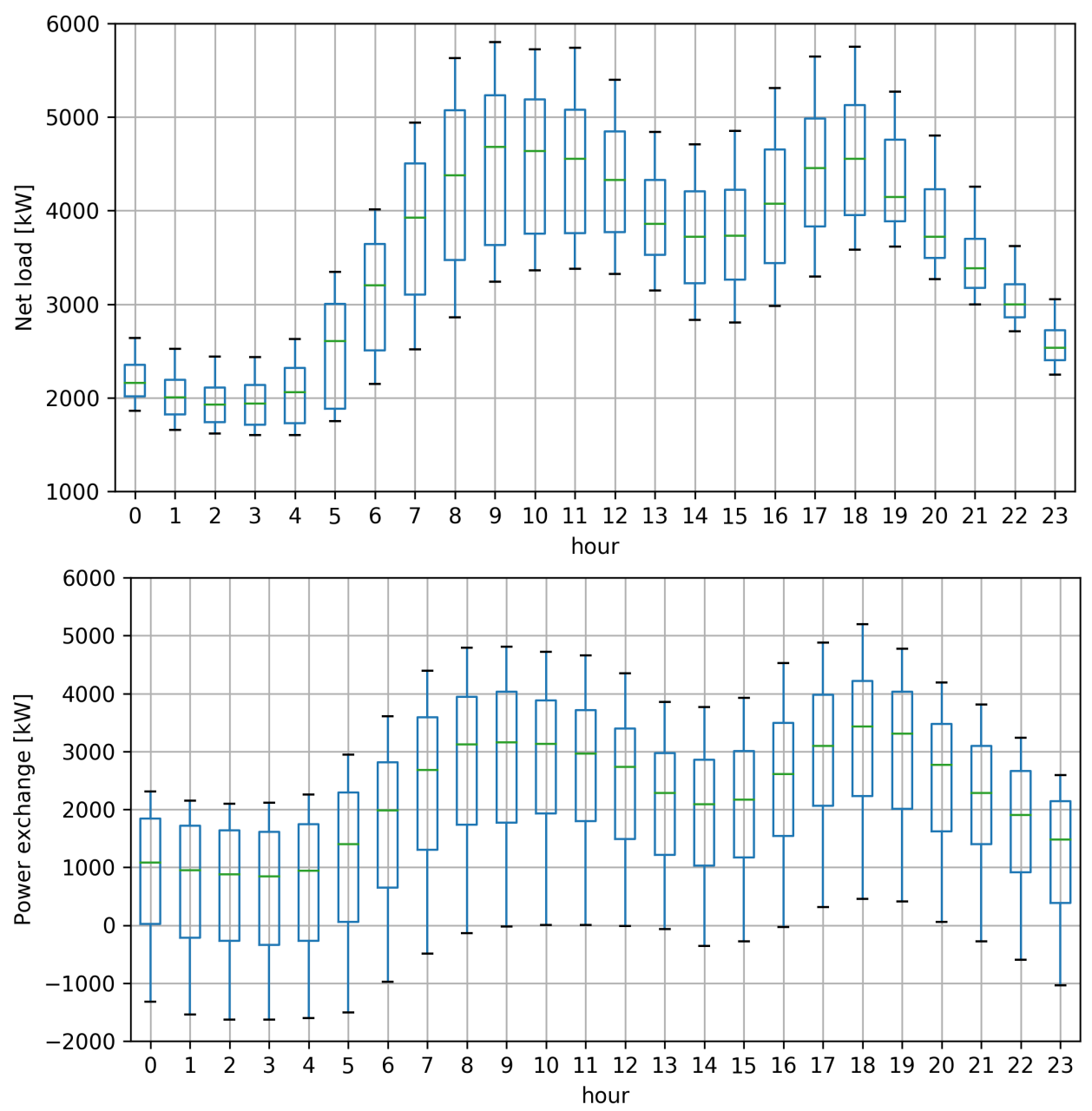

Figure 2. Box plots of the net load and of the power exchange of the microgrid. The box represents the interquartile range, the line in the box is the median, and the whiskers are set at the 5 and 95 percentile of the data range.

\subsection{Weather Forecast}

The weather forecasts of wind speed at $100 \mathrm{~m}$ above ground (height more representative of the wind effect on turbines), radiation, cloud cover, and ground temperature are provided by the European Centre for Medium-Range Weather Forecasts (ECMWF) operating model (OP) [43]. For the three years examined, the OP ECMWF model was the TL1279L137 (horizontal resolution equivalent to about $14 \mathrm{~km}$ at medium latitudes with 137 vertical levels). 
The data were collected from the ECMWF MARS archive for 00GMT analysis and forecast time up to $48 \mathrm{~h}$ with a prediction interval of $1 \mathrm{~h}$. The weather data were interpolated from the values on the nearest four grid points with weights inversely proportional to the distance.

\section{Method}

The power balance of the microgrid can be written as:

$$
p_{e}+p_{g}-p_{n}=0,
$$

in which $p_{e}$ is the net power exchanged by the microgrid (positive in sign if entering in the system), $p_{g}$ is the power produced by the MV generators, and $p_{n}$ is the net load of the low-voltage distribution grid in the island (see Figure 1).

The $p_{g}$ may be divided into components due to the renewable energy generators: $p_{p v}$ is the power due to the PV plant and $p_{w t}$ is the power produced by the wind turbines:

$$
p_{g}=p_{p v}+p_{w t}
$$

The net load can in turn be divided into a pure load component $p_{l}$ and the distributed generation component $p_{d g}$ due to the domestic PV installed on the island:

$$
p_{n}=p_{l}-p_{d g}
$$

Only the measures $p_{e}$ and $p_{n}$ are available, and the task is to obtain a forecast of the exchanged power $\hat{p}_{e}$ and of the net load $\hat{p}_{n}$.

\subsection{Generated Power Forecast}

The power generated by wind turbines and the PV plant is directly related to weather conditions.

A model of the PV power plant has been developed in [44], in which a multivariate adaptive regression spline (MARS) approach is used to derive a regression model that links the power to the forecasts of the Global Horizontal Irradiation (GHI) and of the total cloud cover (TCC) produced by a numerical weather prediction model. The same procedure have been adopted for this analysis.

The wind turbines have been studied in [45], where a distribution mapping (DM) method is presented and used to forecast the power produced by the turbines from the forecast of the wind speed at $100 \mathrm{~m}$ (WS100).

The forecast of the power generated by the MV plants is obtained from Equation (2). Table 2 lists the main properties of this and the other models used for this work. 
Table 2. Models and feature set for each forecast model. The first column represents the distinguishing abbreviation of the model; in the second column, the forecast variable is indicated; in the third column, the predictive technique used; and finally in the fourth column, the predictors used are listed. PV: photovoltaic forecast model; WT: wind turbine forecast model; MVF: forecast system for the medium voltage production; NL: denotes net load models; PE: denotes power exchange models; $\hat{p}_{p v}$ : forecast of MV photovoltaic production; $\hat{p}_{w t}$ : forecast of MV wind turbine production; $\hat{p}_{g}$ : forecast of total MV power production; $\hat{p}_{n}$ : forecast of LV net load; $\hat{p}_{e}$ : forecast of power exchange; MARS: multivariate adaptive regression spline; DM: distribution mapping; SVR: support vector machine regression; GHI: Global Horizontal Irradiation; TCC: total cloud cover; WS100: wind speed at 100 m; T2: forecast of the temperature $2 \mathrm{~m}$ above ground; h: the hour of the day; dow: decimal for the day of the week; doy: days from the beginning of the year; work: indicates whether the day of the measure or forecast is a working day or not (including holidays); dst: indicates whether the time is relative to the period in which daylight saving is active; $p_{n 2 d}$ and $p_{n 7 d}$ : net load 2 days and 7 days before the forecast; $p_{e 2 d}$ and $p_{e 7 d}$ : power exchange 2 days and 7 days before the forecast; $p_{g 2 d}$ and $p_{g 7 d}$ : power generated by the MV plants 2 days and 7 days before the forecast.

\begin{tabular}{cccc}
\hline Model & Variable & Technique & Features \\
\hline PV & $\hat{p}_{p v}$ & MARS & GHI, TCC \\
WT & $\hat{p}_{w t}$ & DM & WS100 \\
MVF & $\hat{p}_{g}$ & Equation (2) & $\hat{p}_{p v}, \hat{p}_{w t}$ \\
\hline NL0 & $\hat{p}_{n}$ & SVR & $p_{n 2 d}, p_{n 7 d}, \mathrm{~h}$, dow, doy, work, dst \\
NL1 & $\hat{p}_{n}$ & SVR & $T 2, p_{n 2 d}, p_{n 7 d}, \mathrm{~h}$, dow, doy, work, dst \\
NL2 & $\hat{p}_{n}$ & SVR & $G H I, p_{n 2 d}, p_{n 7 d}, \mathrm{~h}$, dow, doy, work, dst \\
NL3 & $\hat{p}_{n}$ & SVR, Equations (3), (6) & $\hat{p}_{p v}, p_{n 2 d}, p_{n 7 d}, \mathrm{~h}$, dow, doy, work, dst \\
\hline PE0 & $\hat{p}_{e}$ & SVR & $p_{e 2 d}, p_{e 7 d}, \mathrm{~h}$, dow, doy, work, dst \\
PE1 & $\hat{p}_{e}$ & SVR & GHI, WS100, $p_{e 2 d}, p_{e 7 d,}$ \\
& & & $\mathrm{~h}$, dow, doy, work, dst \\
PE2 & $\hat{p}_{e}$ & SVR & $p_{e 2 d}, p_{e 7 d}, p_{g 2 d}, p_{g 7 d}, p_{n 2 d}, p_{n 7 d}$, \\
PE3 & $\hat{p}_{e}$ & Equation $(7)$ & $\mathrm{h}$, dow, doy, work, dst \\
\hline
\end{tabular}

\subsection{Load Forecast}

The power absorbed by the low-voltage distribution grid is directly linked to the habits of the population of the island, which obviously has a daily, weekly, and yearly periodicity.

Support vector machine regression (SVR) is a widely used and efficient technique for regression in the field; it has been adopted in this analysis due to its efficiency and the relatively small data set that is required for its training.

Several features have been used for the SVR model: the hour of the day, the day of the week, and the day in the year have been used, transforming them as follows to ease the introduction of the cited cyclic behaviors in the model:

$$
\begin{aligned}
\sin _{d} & =\sin (2 \pi h / 24) & \cos _{d} & =\cos (2 \pi h / 24) \\
\sin _{w} & =\sin (2 \pi \text { dow } / 7) & \cos _{w} & =\cos (2 \pi \text { dow } / 7) \\
\sin _{y} & =\sin (2 \pi \text { doy } / 365) & \cos _{y} & =\cos (2 \pi \text { doy } / 365),
\end{aligned}
$$

where $h$ is the hour of the day, dow is a decimal for the day of the week, and doy is the days from the beginning of the year. As tourism seems to have a significant influence on electricity demand, an additional feature work is used, indicating whether the day of the measure or forecast is a working day or not, including holidays. Moreover, the feature $d s t$ indicates whether the time is relative to the period in which daylight saving is active.

All the features have been normalized, and a radial basis function has been chosen as kernel of the SVR algorithm. 
These features alone would allow a decent but unsatisfactory model to be obtained for load prediction. In this case, it is of paramount importance to include the recent history of loading as part of the features considered. Taking into account the 2-day forecast horizon, the loads measured 2 days before and one week before the forecast time have been considered and are denoted as $p_{n 2 d}$ and $p_{n 7 d}$.

$$
p_{n 2 d}(t)=p_{n}(t-2 \text { days }) \quad p_{n 7 d}(t)=p_{n}(t-7 \text { days }) .
$$

A first model was generated using the SVR technique and the feature set just described, and it has been denoted with $N L O$. The main properties of this model and of all the others are listed in Table 2.

To verify the influence of weather on the net load forecast, a similar model was trained using the forecast of the temperature $2 \mathrm{~m}$ above ground (T2) as an additional feature, denoted as NL1.

Since a significant distributed generation from PV panels is present in the distribution grid, an influence of the solar irradiation is expected. A model denoted with NL2 was trained, based on the same feature set as $\mathrm{NLO}$ with the addition of the forecast of the GHI.

Finally, for the net load forecast, another approach is proposed that takes advantage of Equation (3) and is labeled with NL3. Equation (3) separates the contribution of the power demand $p_{l}$ from the distributed generation $p_{d g}$. The latter is not measured directly, but can be estimated by scaling the output of the photovoltaic system according to the ratio between the total installed photovoltaic capacity $(p v c)$ and the peak power of the plant $\left(P V_{\text {peak }}\right)$. The measurement of the power generated by the PV plant is not available for all three years of study, so an estimate is used: $\bar{p}_{p v}$ is obtained, like $\hat{p}_{p v}$, from the regression model for the plant and the weather conditions.

$$
p_{d g} \approx \frac{p v c}{P V_{p e a k}} \bar{p}_{p v}
$$

The assumption of optimal orientation and good maintenance of domestic PV appliances is implicit in this, probably leading to an overestimation of the distributed generation. As mentioned above, the power demand $p_{l}$ is not measured directly. However, it is possible to have an estimate of it from Equations (3) and (6).

An SVR model can then be trained on $p_{l}$ using the same features adopted for model NL0. This SVR model is then used to forecast $\hat{p}_{l}$, Equation (6) is used to obtain $\hat{p}_{d g}$, and finally Equation (3) is used to calculate $\hat{p}_{n}$. See Table 2 for a summary.

\subsection{Power Exchange Forecast}

In order to predict the net power exchange between the island and the mainland's power grid and to assess the influence of weather conditions on its performance, the same incremental approach was used as for net load forecasts. The characteristics of the models are summarized in Table 2.

The first model is labeled with PE0; it is an SVR model that uses a feature set similar to that adopted for $N L 0$, and therefore no weather influence is included. The features $p_{n 2 d}$ and $p_{n 7 d}$ have been replaced by $p_{e 2 d}$ and $p_{e 7 d}$.

The weather forecast is incorporated in the feature set of the second model, labeled as PE1, with the estimates of GHI and WS100.

These two models are blind to the grid structure and details of renewable energy installations. In the third model-labeled with PE2—all the information available for the Borkum grid has been used as features for an SVR model. Included are measures of power exchange, MV generation $\left(p_{g 2 d}\right.$, $\left.p_{g 7 d}\right)$, and LV net load $\left(p_{n 2 d}, p_{n 7 d}\right) 2$ and 7 days before the the time of forecast. The forecast of MV generation by the PV plant and wind turbines are additional features of the model, indirectly including the influence of atmospheric conditions. 
Finally, for model PE3, the Equation (1) is exploited. Separate forecasts for the net load $\hat{p}_{n}$ and for the power generation $\hat{p}_{g}$ are obtained from model NL3 and the PV plant and wind turbines models. The power exchange forecast is obtained algebraically:

$$
\hat{p}_{e}=\hat{p}_{n}-\hat{p}_{g}
$$

\section{Results and Discussion}

\subsection{Load Forecast}

The electrical load prediction for the island of Borkum is complicated by the distributed photovoltaic generation "behind the meter" $(D G)$. The effect of $D G$ on the load introduces a dependence on the weather conditions that overlaps with the typical daily and weekly usage patterns of customers. current use of domestic and commercial electricity can be affected by weather conditions, such as air conditioning during hot summer hours or heating in winter.

In order to investigate the effect of weather conditions on the electrical load, a series of different experiments were conducted, making different hypotheses and using corresponding forecasting approaches, the results of which are summarized in Table 3.

Table 3. Error analysis on the load forecast, from +24 to $+48 \mathrm{~h}$, for different forecast approaches. Row NLO shows the results without taking into account weather influence and using as predictor only statistics about load two and seven days before. Row NL1 refers to the forecast obtained adding as predictor forecast of temperature at $2 \mathrm{~m}$ from the ground; NL2 as before but using as predictor, forecast for GHI instead of T2. The last row shows the results for model NL3, in which a separate model forecasts the photovoltaic distributed generation. The values of nMAE and nRMSE are normalized mean absolute error and root mean squared error with respect to the mean value of the measured quantity.

\begin{tabular}{cccccccc}
\hline Model & $\mathbf{R}^{\mathbf{2}} \mathbf{( - )}$ & MAE (kW) & RMSE (kW) & nMAE (\%) & nRMSE (\%) & Q1 (kW) & Q3 (kW) \\
\hline NL0 & 0.950 & 182.1 & 247.0 & 5.23 & 7.10 & -87.7 & 165.5 \\
NL1 & 0.950 & 182.8 & 247.8 & 5.25 & 7.12 & -87.5 & 167.2 \\
NL2 & 0.955 & 173.2 & 233.7 & 4.97 & 6.71 & -89.9 & 156.6 \\
NL3 & 0.957 & 170.3 & 228.7 & 4.89 & 6.57 & -85.3 & 158.1 \\
\hline
\end{tabular}

Results of the first model NLO-which does not take into account any influence of weather conditions-are shown in the first row of Table 3.

Meteorological conditions may have an impact on electricity consumption [46-48]. It has been shown that the electrical load is low if the ground temperature ranges between 45 and 65 degrees Fahrenheit, while it increases outside this range due to the use of heating and cooling systems. The forecast of temperature $2 \mathrm{~m}$ above surface has been added as a predictor to the approach described above to evaluate the effect of varying weather on the consumption habits. The results in Table 3 row NL1 are almost identical to those for NLO, demonstrating that electrical consumption habits in Borkum are most likely not influenced by outside temperature-at least for a short-term forecast. The temperature effect is probably hidden in the correlation with the power measurements 2 and 7 days before, which are used as predictors for this model. This result is confirmed by the plot in Figure 3, in which the error between the measure and the forecast obtained with model NLO is plotted against the temperature, showing no apparent pattern or correlation (coefficient of determination $\left.\mathrm{R}^{2}=-1.67\right)$. 


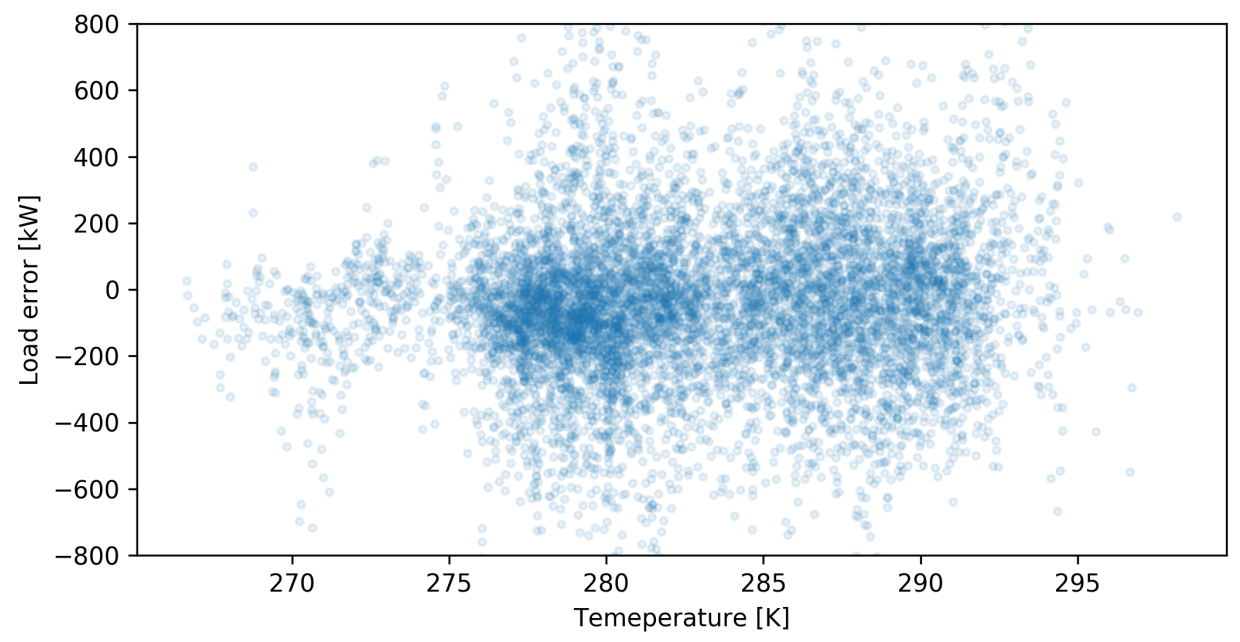

Figure 3. Error in the LV load forecast as a function of the temperature $2 \mathrm{~m}$ above ground forecast. Coefficient of determination $\mathrm{R}^{2}=-1.67$.

Assuming that to be true, the possibility of filtering out the distributed generation from the electric load has been investigated.

First, the ECMWF forecast for GHI is added as predictor, and an SVR model is trained and labeled NL2. The results of Table 3 show a significant improvement, with $R^{2}$ going from 0.950 to 0.955 and and MAE and RMSE decreasing by about 10 and $14 \mathrm{~kW}$, respectively. This demonstrates the importance of solar irradiation for predicting net load through the effect of the distributed generation in the grid.

In the NL3 model, the load of the LV grid $p_{l}$ and the distributed generation $p_{d g}$ are predicted separately. The forecast $\hat{p}_{d g}$ is proportional to the forecast of the MV power plant as for Equation (6), the value of the total installed capacity is known and is equal to $953.2 \mathrm{~kW}$. The errors for this forecast approach are shown in row NL3 of Table 3. They show a further improvement in performance with an increase of $\mathrm{R}^{2}$ from 0.955 to 0.957 and a decrease of mean absolute error (MAE) and root mean squared error (RMSE) of about 3 and $5 \mathrm{~kW}$, respectively.

The model NL3 can be used to obtain an estimate of installed capacity. Several instances of the model have been trained and tested using different $p v c$ values. The errors obtained are shown in Figure 4. Note that the minimum errors have been obtained for values of PV capacity close to the real value. It seems that in our study, distributed generation can in principle be separated from the net load if a representative measurement of PV plant production is available, or if it is possible to produce a reasonably accurate estimate of the PV generation. A reasonable estimate of the total installed capacity can also be obtained.

The results in Table 3 highlight the importance of weather forecast in the case of relevant power production from PV devices and the importance of separating load and generation forecast in order to reduce the overall prediction error. Figure 5 shows the box plots for all models. The reduction of the interquartile range and of the whiskers distance is apparent with the improvement of the methodology. The average values of the first and third quartile of the forecast error (Q1 and Q3) are listed in the last two columns of Table 3. 


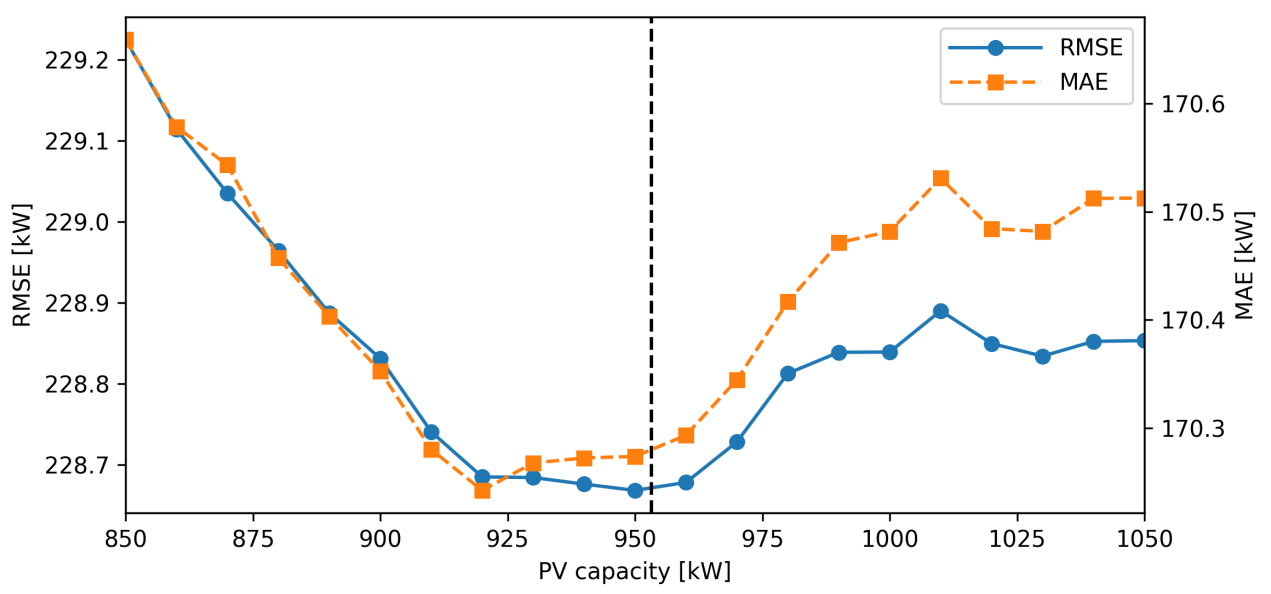

Figure 4. Root mean squared error (RMSE) and mean absolute error (MAE) for load forecast using the approach NL3 as a function of the distributed PV capacity assumed. The vertical line corresponds to the estimated real value obtained by summing up the single utility capacities.
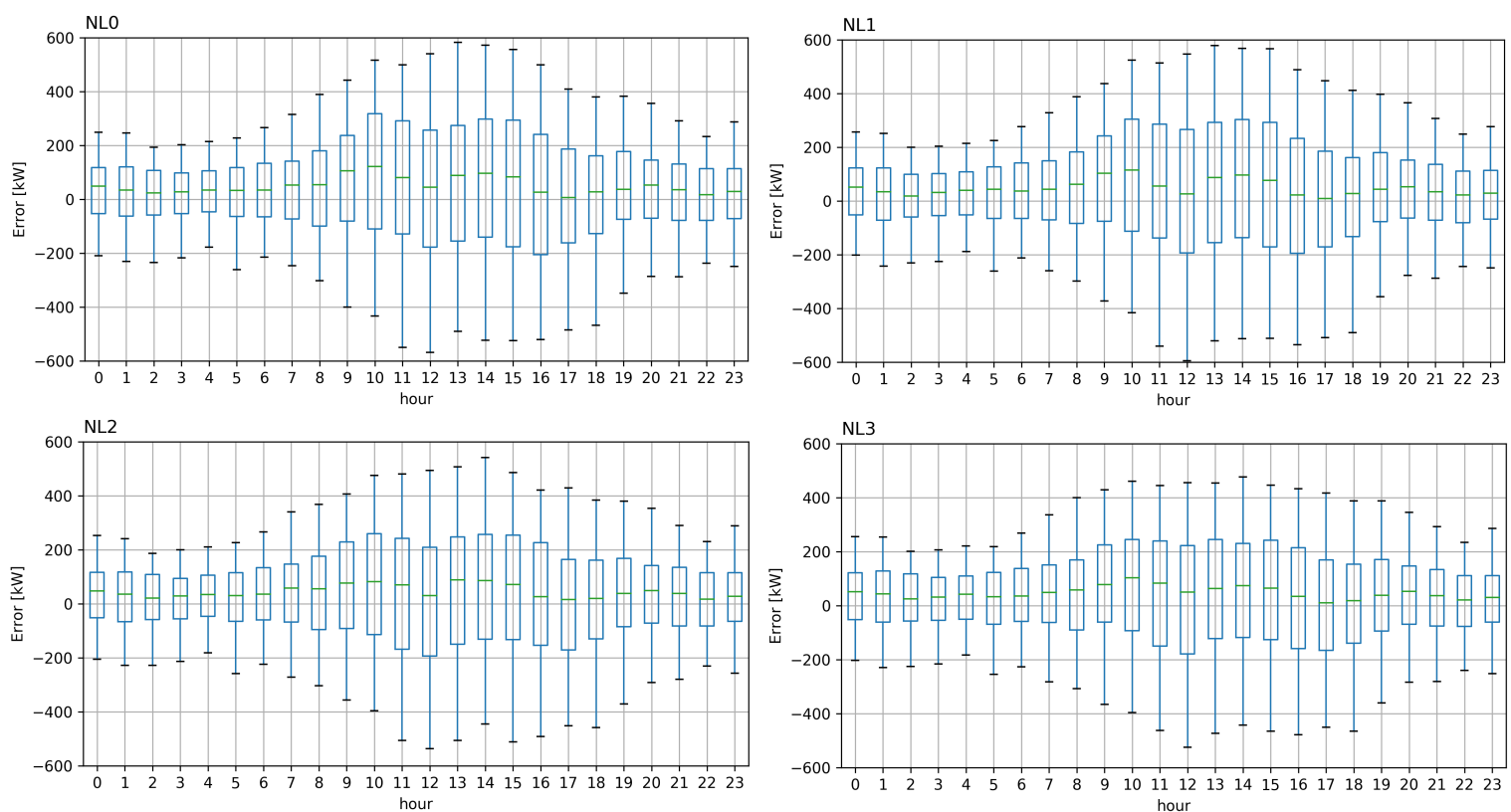

Figure 5. Boxplot of the net load forecast error as a function of the hour of the day. The box represents the interquartile range, the line in the box is the median, and the whiskers are set at the 5 and 95 percentile of the data range. The uncertainty follows the same daily pattern of the PV generation. The improvement in the forecast accuracy obtained with the model NL3 is apparent.

\subsection{Power Exchange Forecast}

In a grid such as that of a small island with high penetration of renewable energies, the balance between generation and load is strongly influenced by the variability of weather conditions. For this reason, an efficient system power exchange of the grid must necessarily incorporate information regarding weather conditions, and at the same time, this must be done in such a way that maximizes the benefits for forecasting.

To do this optimally, some alternative strategies have been studied, using as a benchmark the results obtained by training an SVR learning model using only statistical information extracted from past load observations. The errors obtained with this forecast model PEO are shown in the first row of Table 4 .

Subsequently, the forecasts of GHI and wind speed at $100 \mathrm{~m}$ from the ground are added to the set of features used for $P E 0$, as predictors related to the weather variability. These two additional features 
together should be able to capture the weather influence in net load due to the PV and wind turbine generation. The performance of model PE1 is compared with previous results in Table 4, showing that the influence of weather is substantial and cannot be ignored.

In an attempt to use all available statistical information, another predictor has been built, called PE2, which adds the statistical information available for the measurements of the load and the MV generation to the previous one. Comparison with the PE1 performance shows a substantial improvement in all indicators. $\mathrm{R}^{2}$ increases from 0.784 to 0.864 , and both relative errors of about $7 \%$.

Although all the available information-both as past time series of observed energy flows and as weather forecasts for the atmospheric part-has been used in PE2, even a sophisticated predictor such as SVR may not be able to capture the correct physical form of the production dependence on the weather conditions. However, the latter can be easily incorporated by developing a forecast system for the medium voltage production $(M V F)$ as the sum of the production forecasts for the PV and the two turbines. The detailed features of the MVF system are described in Section 3.1, and the corresponding forecast errors are shown in the first row of Table 4.

Table 4. Error analysis on net load forecast for different prediction strategies. PE1 is the net load forecast obtained using a SVR using as predictors the time series of net load itself and the meteorological forecast for GHI and wind speed at $100 \mathrm{~m}$ from the ground; PE2 is the SVR predictor using all the predictors used for PE1 with the addition of the features used for NL3; PE3 is the net algebraic sum obtained summing the NL3 forecast with the MVF forecast; MVF shows errors of the medium voltage production forecast. The values of nMAE and nRMSE are normalized with respect to the mean value of the measured quantity.

\begin{tabular}{cccccccc}
\hline Model & $\mathbf{R}^{2} \mathbf{( - )}$ & MAE (kW) & RMSE (kW) & nMAE (\%) & nRMSE (\%) & Q1 (kW) & Q3 (kW) \\
\hline MVF & 0.820 & 336.5 & 482.4 & 22.16 & 31.76 & -274.7 & 188.7 \\
PE0 & 0.375 & 971.4 & 1239.3 & 49.50 & 63.15 & -661.0 & 968.6 \\
PE1 & 0.784 & 555.7 & 729.2 & 28.32 & 37.16 & -390.3 & 465.7 \\
PE2 & 0.864 & 429.9 & 577.7 & 21.91 & 29.44 & -296.7 & 356.8 \\
PE3 & 0.880 & 400.7 & 542.8 & 20.42 & 27.66 & -213.7 & 368.9 \\
\hline
\end{tabular}

The model PE3 uses the best forecast for the net load of the low-voltage distribution grid $\hat{p}_{n}$; this is algebraically added to the forecast of the production of the renewable energy plants $\hat{p}_{g}$ to satisfy the power balance.

Errors in the Table 4, show that the latter approach is the most skillful among those considered for this case study, with a significant increase in $\mathrm{R}^{2}$ and a corresponding decrease in nRMSE $(\simeq 1.8 \%)$ and nMAE $(\simeq 1.5 \%)$.

Together with that highlighted for the net load forecast, this result underlines the importance of a correct estimation of the renewable energy generation for the estimation of the net power in a distribution grid. While the model $N L O$ for the low-voltage distribution grid has achieved decent results without including dependence on weather conditions, increasing the supply of energy from renewable sources and introducing power generators that do not have diurnal cycles (e.g., the wind turbine) has a dramatic effect on the quality of the forecast. In fact, the accuracy of model PEO is insufficient, and a good improvement has been obtained by refining the model to PE3.

The results also show-at least in this case-that all information is important for the production of a forecasting model, and that a good understanding of the grid structure and the loads and production systems present—if available—can still offer a significant advantage over a blind machine learning system. Figure 6 confirms this, showing the substantial reduction of the error spread. The average values of Q1 and Q3 are shown in the last columns of Table 4. 

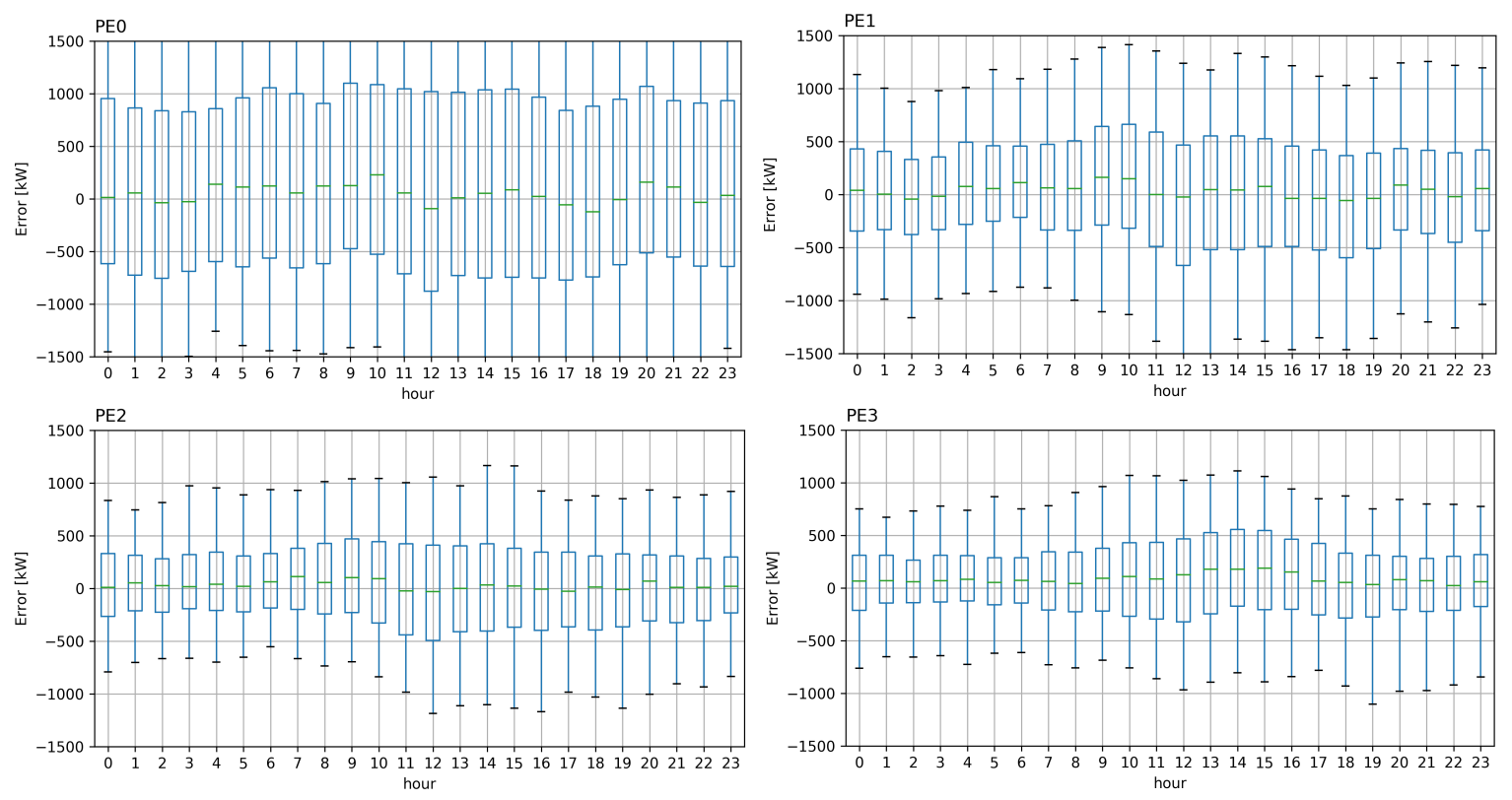

Figure 6. Boxplot of the PE forecast error as a function of the hour of the day. The box represents the interquartile range, the line in the box is the median, and the whiskers are set at the 5 and 95 percentile of the data range. The improvement in the forecast accuracy is evident. The uncertainty is still related to the PV generation, but the pattern is less apparent compared to the net load forecasting case, due to the influence of the wind power forecast uncertainty.

\section{Conclusions}

This study describes and analyzes a forecasting system for the power exchange and the net load of the distribution grid of the German island of Borkum. The island is connected to the power grid by MV cables, but a significant fraction of its energy demand is provided by renewable energy, through distributed generation with domestic PV panels, a medium size PV plant, and two wind turbines.

Several regression models of increasing complexity, based on the support vector machines methodology, have been developed, progressively introducing predictors based on the elaboration of the results of the ECMWF meteorological model.

The forecast horizon is two days, with an hourly resolution.

The results showed that in the case of predicting the net load due to the low voltage distribution grid, there is no significant influence of the ground temperature. The most critical meteorological parameter is represented by radiation to the ground, due to the effect this has on photovoltaic production. In this case, it was possible to obtain a reasonable breakdown between the load and the distributed generation and to make predictive models for the two quantities by having only the net load measurement available. It was also shown how it is possible to obtain a reasonable estimate of the installed photovoltaic power from the measurement of the aggregate net load and a forecasting model for photovoltaic production.

The analysis of the power exchanges in the Borkum grid showed the importance of accurate forecasts for individual renewable production plants as well as for the load. A forecasting model that ignores the network configuration and characteristics of individual systems has failed to achieve the results obtained by making the most of all available information.

The results obtained show that even in the presence of a significant distributed photovoltaic generation, it is still possible to achieve an accurate forecast of the net load. On the contrary, the forecast accuracy for the power exchanged by the island is lower, due to the higher production from renewable 
sources, the concentration of production in single plants of relatively high power, and the difficulty of predicting the generation by wind turbines.

Future developments will be aimed at achieving an improvement in net load forecasting through the introduction of a predictor linked to the tourist presence on the island-for example, using the information related to the hotel bookings or the reservations of seats on the ferries connecting Borkum to the mainland. The prediction of the power exchange of the island's grid will benefit from an improvement in the prediction of the renewable plants' production, for instance by assessing the effectiveness of using the results of the Ensemble Prediction System (EPS) of the ECMWF.

Finally, the effectiveness of the forecasting algorithm will be evaluated when its output is used by the optimal control algorithm of the BESS system that will be installed soon.

Acknowledgments: This work was partially financed by the European Union's Horizon 2020 research and innovation program under grant agreement No 646463, project NETfficient, Energy and economic efficiency for today's smart communities through integrated multi-storage technologies, which also covered the costs to publish in open access, and by the Sardinia Regional Authorities.

Author Contributions: The authors jointly conceived and designed the methodologies, performed the analysis and wrote the paper.

Conflicts of Interest: The authors declare no conflict of interest.

\section{References}

1. Sepasi, S.; Reihani, E.; Howlader, A.M.; Roose, L.R.; Matsuura, M.M. Very short term load forecasting of a distribution system with high PV penetration. Renew. Energy 2017, 106, 142-148.

2. Zhao, H.; Wu, Q.; Hu, S.; Xu, H.; Rasmussen, C.N. Review of energy storage system for wind power integration support. Appl. Energy 2015, 137, 545-553.

3. Yuan, S.; Kocaman, A.S.; Modi, V. Benefits of forecasting and energy storage in isolated grids with large wind penetration-The case of Sao Vicente. Renew. Energy 2017, 105, 167-174.

4. Patra, S.; Kishor, N.; Mohanty, S.R.; Ray, P.K. Power quality assessment in 3-P grid connected PV system with single and dual stage circuits. Int. J. Electr. Power Energy Syst. 2016, 75, 275-288.

5. Kou, P.; Gao, F.; Guan, X. Stochastic predictive control of battery energy storage for wind farm dispatching: Using probabilistic wind power forecasts. Renew. Energy 2015, 80, 286-300.

6. Raza, M.Q.; Nadarajah, M.; Hung, D.Q.; Baharudin, Z. An intelligent hybrid short-term load forecasting model for smart power grids. Sustain. Cities Soc. 2017, 31, 264-275.

7. Sreekumar, S.; Verma, J.; Sujil, A.; Kumar, R. Comparative Analysis of Intelligently Tuned Support Vector Regression Models for Short Term Load Forecasting in Smart Grid Framework. Technol. Econ. Smart Grids Sustain. Energy 2017, 2, 1.

8. Feinberg, E.A.; Genethliou, D. Applied Mathematics for Restructured Electric Power Systems; Chapter-12, "Load Forecasting"; Springer: : New York, NY, USA, 2005; pp. 269-285.

9. Hahn, H.; Meyer-Nieberg, S.; Pickl, S. Electric load forecasting methods: Tools for decision making. Eur. J. Oper. Res. 2009, 199, 902-907.

10. Kyriakides, E.; Polycarpou, M. Short term electric load forecasting: A tutorial. Trends Neural Comput. 2007, 35, 391-418.

11. Muñoz, A.; Sánchez-Úbeda, E.F.; Cruz, A.; Marín, J. Short-term forecasting in power systems: A guided tour. In Handbook of Power Systems II; Springer: New York, NY, USA, 2010; pp. 129-160.

12. Singh, A.K.; Ibraheem; Khatoon, S.; Muazzam, M.; Chaturvedi, D.K. Load forecasting techniques and methodologies: A review. In Proceedings of the IEEE 2nd International Conference on Power, Control and Embedded Systems (ICPCES), Allahabad, India, 17-19 December 2012; pp. 1-10.

13. Mirowski, P.; Chen, S.; Kam Ho, T.; Yu, C.N. Demand forecasting in smart grids. Bell Labs Tech. J. 2014, 18, 135-158.

14. Barak, S.; Sadegh, S.S. Forecasting energy consumption using ensemble ARIMA-ANFIS hybrid algorithm. Int. J. Electr. Power Energy Syst. 2016, 82, 92-104.

15. Hagan, M.T.; Behr, S.M. The time series approach to short term load forecasting. IEEE Trans. Power Syst. 1987, 2, 785-791. 
16. Boroojeni, K.G.; Amini, M.H.; Bahrami, S.; Iyengar, S.; Sarwat, A.I.; Karabasoglu, O. A novel multi-time-scale modeling for electric power demand forecasting: From short-term to medium-term horizon. Electr. Power Syst. Res. 2017, 142, 58-73.

17. Singh, S.K.; Sinha, N.; Goswami, A.K.; Sinha, N. Several variants of Kalman Filter algorithm for power system harmonic estimation. Int. J. Electr. Power Energy Syst. 2016, 78, 793-800.

18. Taylor, J.W. Short-term electricity demand forecasting using double seasonal exponential smoothing. J. Oper. Res. Soc. 2003, 54, 799-805.

19. Bin, H.; Zu, Y.X.; Zhang, C. A Forecasting Method of Short-Term Electric Power Load Based on BP Neural Network. Appl. Mech. Mater. Trans. Tech. Publ. 2014, 538, 247-250.

20. Ghofrani, M.; Ghayekhloo, M.; Arabali, A.; Ghayekhloo, A. A hybrid short-term load forecasting with a new input selection framework. Energy 2015, 81, 777-786.

21. Men, Z.; Yee, E.; Lien, F.S.; Wen, D.; Chen, Y. Short-term wind speed and power forecasting using an ensemble of mixture density neural networks. Renew. Energy 2016, 87, 203-211.

22. Li, S.; Goel, L.; Wang, P. An ensemble approach for short-term load forecasting by extreme learning machine. Appl. Energy 2016, 170, 22-29.

23. Vaz, A.; Elsinga, B.; van Sark, W.; Brito, M. An artificial neural network to assess the impact of neighbouring photovoltaic systems in power forecasting in Utrecht, the Netherlands. Renew. Energy 2016, 85, 631-641.

24. Li, Y.; Wen, Z.; Cao, Y.; Tan, Y.; Sidorov, D.; Panasetsky, D. A combined forecasting approach with model self-adjustment for renewable generations and energy loads in smart community. Energy 2017, 129, $216-227$.

25. Amarasinghe, K.; Marino, D.L.; Manic, M. Deep neural networks for energy load forecasting. In Proceedings of the IEEE 26th International Symposium on Industrial Electronics (ISIE), Edinburgh, UK, 19-21 June 2017; pp. 1483-1488.

26. Vapnik, V.; Golowich, S.E.; Smola, A.J. Support vector method for function approximation, regression estimation and signal processing. In Proceedings of the 9th International Conference on Advances in Neural Information Processing Systems, Denver, CO, USA, 3-5 December 1996; pp. 281-287.

27. Hong, W.C. Electric load forecasting by support vector model. Appl. Math. Model. 2009, 33, 2444-2454.

28. Humeau, S.; Wijaya, T.K.; Vasirani, M.; Aberer, K. Electricity load forecasting for residential customers: Exploiting aggregation and correlation between households. In Proceedings of the IEEE Sustainable Internet and ICT for Sustainability (SustainIT), Palermo, Italy, 30-31 October 2013; pp. 1-6.

29. Chen, B.J.; Chang, M.W.; Lin, C.J. Load forecasting using support vector machines: A study on EUNITE competition 2001. IEEE Trans. Power Syst. 2004, 19, 1821-1830.

30. Niu, D.; Dai, S. A Short-Term Load Forecasting Model with a Modified Particle Swarm Optimization Algorithm and Least Squares Support Vector Machine Based on the Denoising Method of Empirical Mode Decomposition and Grey Relational Analysis. Energies 2017, 10, 408.

31. Marčiukaitis, M.; Žutautaite, I.; Martišauskas, L.; Jokšas, B.; Gecevičius, G.; Sfetsos, A. Non-linear regression model for wind turbine power curve. Renew. Energy 2017, 113, 732-741.

32. Jung, J.; Broadwater, R.P. Current status and future advances for wind speed and power forecasting. Renew. Sustain. Energy Rev. 2014, 31, 762-777.

33. Antonanzas, J.; Osorio, N.; Escobar, R.; Urraca, R.; Martinez-de Pison, F.; Antonanzas-Torres, F. Review of photovoltaic power forecasting. Sol. Energy 2016, 136, 78-111.

34. Kaur, A.; Pedro, H.T.; Coimbra, C.F. Impact of onsite solar generation on system load demand forecast. Energy Convers. Manag. 2013, 75, 701-709.

35. Haupt, S.E.; Dettling, S.; Williams, J.K.; Pearson, J.; Jensen, T.; Brummet, T.; Kosovic, B.; Wiener, G.; McCandless, T.; Burghardt, C. Blending distributed photovoltaic and demand load forecasts. Sol. Energy 2017, 157, 542-551.

36. Reihani, E.; Sepasi, S.; Roose, L.R.; Matsuura, M. Energy management at the distribution grid using a Battery Energy Storage System (BESS). Int. J. Electr. Power Energy Syst. 2016, 77, 337-344.

37. Jin, L.; Cong, D.; Guangyi, L.; Jilai, Y. Short-term net feeder load forecasting of microgrid considering weather conditions. In Proceedings of the IEEE International Energy Conference (ENERGYCON), Dubrovnik, Croatia, 13-16 May 2014; pp. 1205-1209.

38. Kaur, A.; Nonnenmacher, L.; Coimbra, C.F. Net load forecasting for high renewable energy penetration grids. Energy 2016, 114, 1073-1084. 
39. Fonte, P.; Monteiro, C. Net load forecasting in presence of renewable power curtailment. In Proceedings of the IEEE 13th International Conference on the European Energy Market (EEM), Porto, Portugal, 6-9 June 2016; pp. 1-5.

40. Shaker, H.; Zareipour, H.; Wood, D. Estimating power generation of invisible solar sites using publicly available data. IEEE Trans. Smart Grid 2016, 7, 2456-2465.

41. Zhang, X.; Grijalva, S. A Data-Driven Approach for Detection and Estimation of Residential PV Installations. IEEE Trans. Smart Grid 2016, 7, 2477-2485.

42. Wang, Y.; Zhang, N.; Chen, Q.; Kirschen, D.S.; Li, P.; Xia, Q. Data-Driven Probabilistic Net Load Forecasting with High Penetration of Invisible PV. IEEE Trans. Power Syst. 2017, doi:10.1109/TPWRS.2017.2762599.

43. Persson, A. User Guide to ECMWF Forecast Products; Meteorological Bulletin 3; ECMWF: Reading, UK, 2001.

44. Massidda, L.; Marrocu, M. Use of Multilinear Adaptive Regression Splines and numerical weather prediction to forecast the power output of a PV plant in Borkum, Germany. Sol. Energy 2017, 146, 141-149.

45. Marrocu, M.; Massidda, L. A simple and effective approach for the prediction of turbine power production from wind speed forecast. Energies 2017, 10, 1967.

46. Hor, C.L.; Watson, S.J.; Majithia, S. Analyzing the impact of weather variables on monthly electricity demand. IEEE Trans. Power Syst. 2005, 20, 2078-2085.

47. Drezga, I.; Rahman, S. Input variable selection for ANN-based short-term load forecasting. IEEE Trans. Power Syst. 1998, 13, 1238-1244.

48. Ruth, M.; Lin, A.C. Regional energy demand and adaptations to climate change: Methodology and application to the state of Maryland, USA. Energy Policy 2006, 34, 2820-2833.

(C) 2017 by the authors. Licensee MDPI, Basel, Switzerland. This article is an open access article distributed under the terms and conditions of the Creative Commons Attribution (CC BY) license (http:// creativecommons.org/licenses/by/4.0/). 